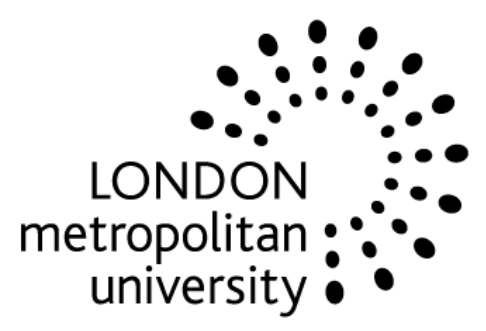

Centre for International Capital Markets

Discussion Papers

ISSN 1749-3412

Multiple Cyclical Fractional Structures in Financial Time Series

Guglielmo Maria Caporale and Luis A. Gil-Alana

No 2008-4 


\title{
MULTIPLE CYCLICAL FRACTIONAL STRUCTURES IN FINANCIAL TIME SERIES
}

\author{
Guglielmo Maria Caporale \\ Brunel University, London
}

\author{
Luis A. Gil-Alana \\ University of Navarra
}

\section{January 2008}

\begin{abstract}
This paper analyses multiple cyclical structures in financial time series. In particular, we focus on the monthly structure of the Nasdaq, the Dow Jones and the Standard\&Poor stock market indices. The three series are modelled as long-memory processes with poles in the spectrum at multiple frequencies, including the long-run or zero frequency.

JEL Classification: $\mathrm{C} 22$
\end{abstract}

Keywords: Multiple cyclical structures, Long memory, Stock market indices.

Corresponding author: Professor Guglielmo Maria Caporale, Centre for Empirical Finance, Brunel University, Uxbridge, Middlesex UB8 3PH, UK. Tel.: +44 (0)1895 266713. Fax: +44 (0)1895 269770. Email: Guglielmo-Maria.Caporale@brunel.ac.uk Tecnologia (SEJ2005-07657, Spain). 


\section{Introduction}

Modelling stock market prices is still a controversial matter. While the efficiency market hypothesis suggests that they should follow a random walk (see Fama, 1970; Summers, 1986), other authors have found evidence of mean reversion in their behaviour (see, e.g., Poterba and Summers, 1988 and Fama and French, 1988). The standard econometric approach to settle this issue empirically relies on establishing the (integer) order of integration of the series by carrying out nonstationary unit root tests. More recently, however, the possibility of fractional orders of integration has also been taken into account, implying that the time series may revert to its mean, but at a very slow hyperbolic rate. This idea is implicit in the paper by Caporale and Gil-Alana (2007), where the stochastic process followed by stock prices is decomposed into a long-run component described by the fractional differencing parameter (d) and a shortrun (ARMA) structure. Specifically, assuming that $y_{t}$ is the time series we observe, the stochastic process is assumed to take the following form:

$$
(1-L)^{d} x_{t}=u_{t}, \quad t=1,2, \ldots
$$

where $d$ can be any real number and $u_{t}$ is an $\mathrm{I}(0)$ process, modelled, for instance, as an $\operatorname{ARMA}(\mathrm{p}, \mathrm{q})$ structure. Processes such as (1) are characterised by a spectral density function which is unbounded at the zero frequency, while the remaining frequencies are positive and bounded. In this paper we extend this model by considering more general processes which may contain more than a single pole or singularity at the spectrum. In such a case, the autocorrelation function decays hyperbolically as in (1) but in a much more complicated way; this was suggested by Robinson (2001) as an alternative way of describing the behaviour of many financial time series. 


\section{The statistical model}

Let us consider the following process,

$$
\prod_{r=1}^{k}\left(1-2 \cos w_{j_{r}} L+L^{2}\right)^{d_{r}} y_{t}=u_{t}, \quad t=1,2, \ldots
$$

where $\mathrm{k}$ is a finite integer indicating the maximum number of cyclical structures; $w_{j_{r}}=2 \pi / j_{r}$ where $\mathrm{j}_{\mathrm{r}}$ indicates the number of time periods per cycle corresponding to the $\mathrm{r}^{\text {th }}$ cyclical structure, $\mathrm{L}$ is the lag operator (i.e., $\mathrm{Ly}_{\mathrm{t}}=\mathrm{y}_{\mathrm{t}-1}$ ), and $\mathrm{u}_{\mathrm{t}}$ is an $\mathrm{I}(0)$ process assumed to be covariance-stationary with spectral density function that is positive and finite at any frequency. Note that each cyclical structure $\left(1-2 \cos w_{j_{r}} L+L^{2}\right)^{d_{r}}$ represents a Gegenebauer process and, defining $\mu_{\mathrm{r}}=\cos \left(\mathrm{w}_{\mathrm{r}}\right)$, it can be computed recursively as

$$
\begin{aligned}
& C_{0, d_{r}}\left(\mu_{r}\right)=1, C_{1, d_{r}}\left(\mu_{r}\right)=2 \mu_{r} d_{r}, \text { and } \\
& C_{j, d_{r}}\left(\mu_{r}\right)=2 \mu_{r}\left(\frac{d_{r}-1}{j}+1\right) C_{j-1, d_{r}}\left(\mu_{r}\right)-\left(2 \frac{d_{r}-1}{j}+1\right) C_{j-2, d_{r}}\left(\mu_{r}\right), \quad j=2,3, \ldots .
\end{aligned}
$$

(see, among others, Magnus et al., 1966, or Rainville, 1960, for further details on Gegenbauer polynomials). Gray et al. (1989) showed that $y_{t}$ in (2) is stationary (with respect to the $\mathrm{r}^{\text {th }}$ frequency) if $d_{r}<0.5$ for $\left|\mu_{r}\right|<1$ and if $d_{r}<0.25$ for $\left|\mu_{r}\right|=1$. $^{1}$

The specification given by (2) can be tested by using a version of the parametric procedure of Robinson (1994), noting that the long-run polynomial $(1-L)^{d_{1}}$ can also be expressed as a cyclical polynomial with $\mathrm{j}_{1}=1$. $^{2}$ This method, which is based on the

\footnotetext{
${ }^{1}$ Lobato and Robinson (1998) proposed a semiparametric approach for testing this type of model, and Dalla and Hidalgo (2005) suggested a parametric test where the unbounded frequency in the spectrum is assumed to be unknown.

${ }^{2}$ Note that, if $\mathrm{j}_{1}=1$, then $\left(1-2 \cos w_{j_{1}} L+L^{2}\right)^{d_{1}}=\left(1-2 L+L^{2}\right)^{d_{1}}=(1-L)^{2 d_{1}}$.
} 
Lagrange Multiplier (LM) principle, will be applied in the following section to three US stock market indices.

\section{Empirical Analysis}

The series analysed in this section are the three most common US stock market indices, i.e., the Nasdaq, the Dow Jones and the Standard\&Poor indices, monthly. The starting dates are January 1971 (Nasdaq), February 1951 (Dow Jones) and February 1964 (Dow Jones), and the sample ends in October 2007.

Initially we assume that there are three cyclical structures underlying the process and consider a model of the form:

$$
\prod_{r=1}^{3}\left(1-2 \cos w_{j_{r}} L+L^{2}\right)^{d_{r}} y_{t}=u_{t}, \quad t=1,2, \ldots
$$

with white noise $\mathrm{u}_{\mathrm{t}}$, testing the following null hypothesis:

$$
d=\left(d_{1}, d_{2}, d_{3}\right)^{\prime}=\left(d_{1 o}, d_{2 o}, d_{3 o}\right)^{\prime}=d_{o},
$$

for $\mathrm{d}_{0}$-values running from 0 to 2 with 0.01 increments, and $\left(\mathrm{j}_{\mathrm{r}}\right)_{\mathrm{r}=1,2,3}$ equal to $1,2, \ldots$, $\mathrm{T} / 2$ with $\mathrm{j}_{1} \neq \mathrm{j}_{2} \neq \mathrm{j}_{3}$. Then, we estimate the $\mathrm{j}$ 's and the $d$ 's by choosing the value that minimises the test statistic in absolute value. ${ }^{3}$ The results for the 3 -cycle case are reported in Table 1.

\section{INSERT TABLE 1 ABOUT HERE}

First of all, it is noteworthy that for the three series the first cycle corresponds to $\mathrm{j}_{1}=1$, which is precisely the long-run or zero frequency, and the associated orders of integration at this frequency are 0.38 for the Nasdaq, and around 0.5 for the Dow Jones

\footnotetext{
${ }^{3}$ Note that Robinson's (1994) method is based on the Whittle function, which is an approximation of the likelihood function.
} 
and the Standard\&Poor. Note that these values should be multiplied by two to obtain the proper orders of integration at the long-run frequency (see footnote 2), implying that the Nasdaq index is mean-reverting at this particular frequency. Focusing now on the second frequency, one can see that $\mathrm{j}_{2}=23$ for the Nasdaq, 12 for the Dow Jones and 4 for the Standard\&Poor, implying that the cycles repeat themselves every two years for the Nasdaq, yearly for the Dow Jones, and quarterly for the Standard\&Poor index. The third cycle has a periodicity of about 5 years for the three indices and, although $d_{3}$ is above zero in the three cases, the confidence intervals include zero, implying that this frequency can be discarded. Thus, next we perform the procedure using only two cyclical structures for each series. The results are reported in Table 2.

\section{INSERT TABLE 2 ABOUT HERE}

One can see that the first cyclical component again corresponds to the long-run frequency $\left(\mathrm{j}_{1}=1\right)$, while the second cycle has now a periodicity of 55 periods for the Nasdaq, 60 for the Standard\&Poor, and 80 for the Dow Jones. This cyclical component may be related to the business cycle frequencies underlying the series. With respect to the long-run frequency, the order of integration (multiplied by two) is slightly below unity for the Nasdaq and the Standard\&Poor, and it is slightly above 1 for the Dow Jones. The second cyclical structure, possibly related to its business cycle characteristics, is positive but smaller than 0.5 , implying long memory but stationarity in the three indices. 


\section{Conclusions}

In this paper we have examined the existence of multiple cyclical structures in the context of long-range dependence in various stock market indices. In particular, we have proposed a general specification that allows for more than a single pole or singularity at the spectrum. The empirical application to three US stock market indices suggests that there might exist two components with long-memory behaviour. The first one appears to be related to the long-run behaviour and the order of integration is about one in the three cases. The second one is probably related to the business cycle characteristics of the series since the periodicity seems to repeat itself every 5 or 6 years, and the order of integration is below 0.5 implying stationarity with respect to this component. Therefore, according to this specification shocks affecting the long-run component are long-lived and possibly permanent in case of the Dow Jones index, while shocks affecting the cyclical structure are mean reverting, disappearing in the long run.

\section{References}

Caporale, G.M. and L.A. Gil-Alana (2007) Long run and cyclical dynamics in the US stock market, CESifo Working Paper no. 2046.

Dalla, V. and J. Hidalgo (2005) A parametric bootstrap test for cycles. Journal of Econometrics 129, 219-261.

Fama, E.F. (1970) Efficient capital markets: a review of theory and empirical work, Journal of Finance 25, 383-417.

Fama, E.F. and K.R. French (1988) Permanent and transitory components of stock prices, Journal of Political Economy 96, 246-273.

Gray, H.L., Yhang, N. and W.A. Woodward (1989) On generalized fractional processes. Journal of Time Series Analysis 10, 233-257.

Lobato, I. and P.M. Robinson (1998) A non-parametric test for I(0). Review of Economics and Statistics 65, 475-495. 
Magnus, W., Oberhettinger, F. and R.P. Soni (1966) Formulas and theorems for the special functions of mathematical physics. Springer, Berlin.

Poterba, J.M. and L.H. Summers (1988) Mean reversion in stock prices: evidence and implications, Journal of Financial Economics 22, 27-59.

Rainville, E.D. (1960) Special functions, MacMillan, New York.

Robinson, P.M. (1994) Efficient tests of nonstationary hypotheses. Journal of the American Statistical Association 89, 1420-1437.

Robinson, P.M. (2001) The memory of stochastic volatility models, Journal of Econometrics 101, 195-218.

Summers, L.H. (1986) Does the stock market rationally reflect fundamental values?, Journal of Finance 41, 591-601. 


\begin{tabular}{|c|c|c|c|}
\hline \multicolumn{4}{|c|}{ TABLE 1 } \\
\hline \multirow{3}{|c|}{ Estimates in a model with three cyclical structures } \\
\hline \multirow{3}{*}{ Nasdaq } & $\mathrm{j}_{1}=1$ & $\mathrm{j}_{2}=23$ & $\mathrm{j}_{3}=64$ \\
\cline { 2 - 4 } & 0.38 & 0.28 & 0.03 \\
& {$[0.35,0.42]$} & {$[0.20,0.37]$} & {$[0.00,0.08]$} \\
\hline \multirow{3}{*}{ Dow Jones } & $\mathrm{j}_{1}=1$ & $\mathrm{j}_{2}=12$ & $\mathrm{j}_{3}=64$ \\
\cline { 2 - 4 } & 0.50 & 0.50 & 0.10 \\
& {$[0.42,0.88]$} & {$[0.39,0.92]$} & {$[0.00,0.48]$} \\
\hline \multirow{3}{*}{ Standard\&Poor } & $\mathrm{j}_{1}=1$ & $\mathrm{j}_{2}=4$ & $\mathrm{j}_{3}=66$ \\
\cline { 2 - 4 } & 0.49 & 0.90 & 0.01 \\
& {$[0.46,0.53]$} & {$[0.67,1.17]$} & {$[-0.02,0.05]$} \\
\hline
\end{tabular}

In brackets the $95 \%$ confidence band

\section{TABLE 2}

\section{Estimates in a model with two cyclical structures}

\begin{tabular}{|c|c|c|}
\hline \multirow{3}{*}{ Nasdaq } & $\mathrm{j}_{1}=1$ & $\mathrm{j}_{2}=55$ \\
\cline { 2 - 3 } & 0.40 & 0.20 \\
& {$[0.35,0.54]$} & {$[0.11,0.34]$} \\
\hline \multirow{3}{*}{ Dow Jones } & $\mathrm{j}_{1}=1$ & $\mathrm{j}_{2}=80$ \\
\cline { 2 - 3 } & 0.52 & 0.22 \\
& {$[0.44,0.57]$} & {$[0.12,0.27]$} \\
\hline \multirow{3}{*}{ Standard\&Poor } & $\mathrm{j}_{1}=1$ & $\mathrm{j}_{2}=60$ \\
\cline { 2 - 3 } & 0.45 & 0.12 \\
& {$[0.42,0.51]$} & {$[0.05,0.31]$} \\
\hline
\end{tabular}

In brackets the $95 \%$ confidence band 